\title{
A Circularly Polarized Low-Cost Flat Panel Antenna Array with a High Impedance Surface Meta-Substrate for Satellite On-The-Move Medical IoT Applications
}

\author{
Benjamin J. Falkner, Student Member, IEEE, Hengyi Zhou, Amit Mehta, Senior Member, IEEE, Thanos \\ Arampatzis, Dariush Mirshekar-Syahkal, Fellow, IEEE and Hisamatsu Nakano, Life Fellow, IEEE
}

\begin{abstract}
A $1 \times 3$ linear antenna array consisting of Quad-Arm Curl antenna with a High impedance meta-surface $(\mathrm{QACH})$ is presented. We believe that it is the first linear phased array solution which can provide $360^{\circ}$ azimuth coverage. This array has been designed to operate at L-Band (1.518 - 1.675 GHz) and generate right hand circularly polarized radiation to primarily target the Inmarsat BGAN satellite constellation. The metamaterial structure integrated into each antenna element allows a low-profile height of $17.2 \mathrm{~mm}\left(\lambda_{1.597} / 10.9\right)$. Since the curl element has wideband characteristics, the array is able to provide shared aperture functionality. The array guarantees high gain beam steering for low elevation angles (up to $\theta=7^{\circ}$ from the zenith) with an average gain of $7.96 \mathrm{dBic}$ at $\theta=70^{\circ}$. In comparison, to achieve an equivalent high gain a conventional $4 \times 5$ patch array would be required ( 3 elements vs 20 elements). This means that the proposed array requires $80 \%$ fewer phase shifters, amplifiers and LNAs. This translates to a crucial commercial advantage in relation to manufacturing cost. This development can lead to disruption of the existing Satcom market by lowering the barrier-to-entry for customers looking for a mass deployable, low-cost IoT on Satcom solution.
\end{abstract}

Index Terms - Antenna Arrays, Beam Steering, Curl Antenna, Metamaterials, Phased Arrays, Satellite communication

\section{INTRODUCTION}

$\mathrm{R}$ ELIABLE connectivity is vital to IoT applications where an interrupted connection can have implications for system precision, effectiveness and security. On-the-move IoT devices often rely on cellular networks [1] which, while providing low-cost connectivity over a wide area, suffers from regions with little or no coverage [2]. Hence, there is demand for a system that provides reliable communication with global coverage. For this reason, global satellite communication is a natural fit for reliable and un-interrupted mobile connectivity.

The Inmarsat Broadband Global Area Network (BGAN) satellite constellation provides high speed internet access and nearglobal coverage [3-5]. It is an ideal candidate for device-to-cloud connectivity, especially for IoT on-the-move applications. This is because its services are in L-band and are relatively cheap, certified for medical applications and, importantly, it is fully functional today. High speed LEO constellations such as those of SpaceX Starlink and Amazon are yet to be completed [6]. Due to the geostationary (GEO) orbit of the BGAN satellite constellation, a beam steering range of $20^{\circ}<\theta<70^{\circ}$ is required for most of Europe, Australia, China, India and the United States [4]. For data rates of $340 \mathrm{kbps}$, the BGAN terminals must have a gain greater than 6.5

This work is part funded by a grant from the European Space Agency (ESA) (Lightbar project). Web link: https://artes.esa.int/projects/lightbar

B. J. Falkner, H. Zhou and A. Mehta are with the Electrical and Electronic Engineering Department at Swansea University, Fabian Way, Skewen, Swansea, SA1 8EN (email: b.j.falkner@swansea.ac.uk; h.zhou@swansea.ac.uk; a.mehta@swansea.ac.uk)

T. Arampatzis is with Satellite Applications Catapult, Electron Building, Fermi Ave, Didcot, OX11 0QR (email: Thanos.Arampatzis@sa.catapult.org.uk)

D. Mirshekar-Syahkal is with the School of Computer Science and Electronic Engineering (CSEE) at University of Essex, Wivenhoe Park, Colchester CO4 3SQ (email: dariush@essex.ac.uk)

H. Nakano is with the Department of Electrical and Electronics, Science and Engineering at Hosei University, Tokyo, 184-8584, Japan (email: hymat@hosei.ac.jp)
dBic. However, steering to low elevation angles with this gain is a significant challenge for on-the-move satellite terminals, both from a technological and commercial perspective.

Many existing commercial BGAN vehicular on-the-move antennas uses mechanical movement of the antenna to steer the beam to high elevation angles in order to maintain directive gain $[7,8]$. Unfortunately, these antenna systems are typically large, costly, and prone to wear due to their mechanical components. Purely electronic alternatives have been proposed [9,10]. For example, an array designed for the purpose of BGAN was presented in 2011 [11] which was able to provide the required bandwidth and Right Hand Circularly Polarized (RHCP) beam steering down to $40^{\circ}$ elevation. This was achieved using a $4 \times 5$ array of $61 \mathrm{~mm}$ diameter patches. However, as mentioned above, the limited beam steering is not suitable for BGAN communication for much of the earth's surface. Furthermore, the feed circuit for such an array would require a total of 40 phase shifters and 20 High Power Amplifiers (HPA), Low Noise Amplifiers (LNAs) and duplexers. With so many components required, the cost of the feednetwork will raise the price of the overall system significantly.

Additionally, other systems and structures have been developed [12-14] that provide functionality such as wideband coverage and wide steering, albeit with moderately high complex feeding network. They deliver many of the antenna functionalities that are required of the BGAN communication. However, none of them can deliver all of the required BGAN functions simultaneously at a low cost, especially pertaining to producing a $\mathrm{CP}$ beam steering at low elevation angles. A full comparison of these designs with the work presented here is displayed in Table 1 in Section IV.

As a solution to these challenges, we present a $1 \times 3$ linear array consisting of Quad-Arm Curl antennas with a High Impedance Surface (referred to as ' $\mathrm{QACH}$ ') that is capable of providing wideband coverage and high gain beam steering covering elevation angles of $20^{\circ}<\theta<70^{\circ}$ whilst only using a fraction of the components of a typical square phased array. The ability for a linear array to provide complete azimuth steering is one of the key innovations of this work.

The proposed quad-arm curl antenna unit element (Fig. 1) can cover a wide operating bandwidth and hence allows shared aperture functionality. A High Impedance Surface (HIS) layer has been inserted into the quad-arm curl antenna element to provide a $54 \%$ reduction in height from previous designs [15] while also optimizing the antenna performance.

This paper also proposes a hybrid feeding network to excite the array (Fig. 7). The entire feeding system requires only 4 phase shifters, 3 HPAs, 3 LNAs, 3 duplexers and 3 switches for both the $\mathrm{Rx}$ and $\mathrm{Tx}$ bands. This underpins a major reduction in the number of components required in comparison with feeding networks of typical planar arrays [6]. In practical terms, this represents a cost saving of over $75 \%$, providing opportunity for commercialization of low-cost large scale IOT systems. In this regard, a prime example of the applications of this work is IOT for medical ambulances. 
II. QUAD-ARM CURL ANTENNA WITH HIS (QACH)

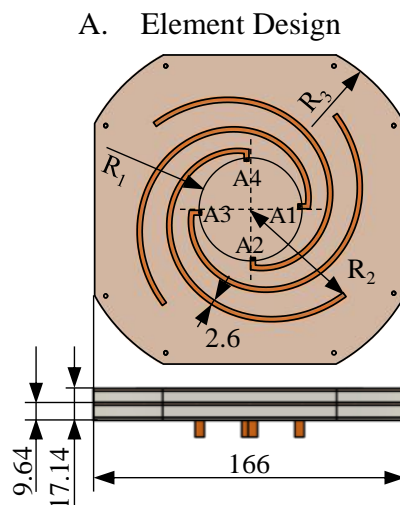

(a)

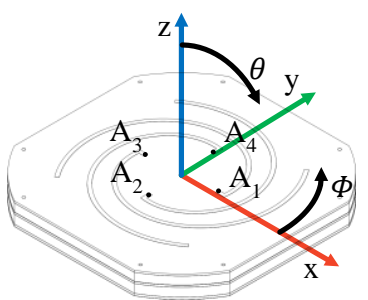

(c)

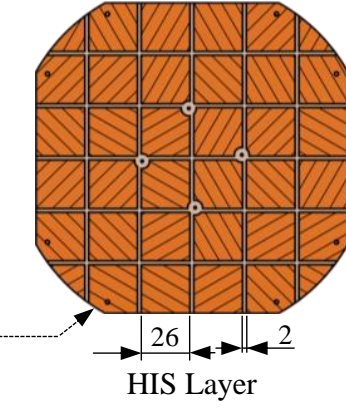

(b)

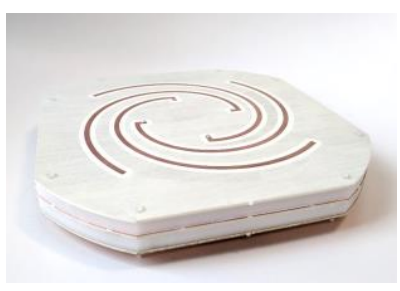

(d)
Fig. 1. QACH element: (a) Antenna geometry top and side view, (b) HIS configuration, (c) Isometric view (with ports and coordinates) and (d) Fabricated prototype. All units are in $\mathrm{mm}$.

The Quad-Arm Curl antenna with HIS (QACH) is constructed of five layers (net height $=17.14 \mathrm{~mm}$ ), as displayed in Fig 1 (a). The antenna element has a width of $166 \mathrm{~mm}$ and beveled corners of $\mathrm{R}_{3}=95 \mathrm{~mm}$ to allow for rotation in the array as presented in Section IV. The curl strip has an inner radius of $\mathrm{R}_{1}=26.59 \mathrm{~mm}$, an outer radius of $\mathrm{R}_{2}=68.28 \mathrm{~mm}$ and a strip width of $2.6 \mathrm{~mm}$. On the top layer, the curl antenna microstrips are printed on $1.5 \mathrm{~mm}$ thick Rogers RO4035B substrate $\left(\varepsilon_{r}=3.48\right.$ and $\left.\delta=0.0037\right)$. Below this is a low-cost $6 \mathrm{~mm}$ thick layer of Delrin plastic $\left(\varepsilon_{\mathrm{r}}=2.6\right.$ at 1.597 $\mathrm{GHz}$ and $\delta=0.005$ ). Delrin plastic has a far lower cost than an equivalent thickness of Rogers RO4035B or FR-4, hence it is used here for constructing the majority of the antenna height. The central layer is a planar HIS as shown in Fig. 1 (b) which is also printed on Rogers RO4035B of $1.5 \mathrm{~mm}$ in thickness. Again, Delrin layer with a height $6.5 \mathrm{~mm}$ is used as a thick substrate below this. Finally, a ground layer made from single sided copper plated FR-4 $\left(\varepsilon_{\mathrm{r}}=4.8\right.$ at $1.597 \mathrm{GHz}$ and $\delta=0.025$ ) of thickness $1.6 \mathrm{~mm}$ is placed at the bottom of the structure. Copper plated FR-4 is used as a ground layer due to its low cost and weight compared to a standalone copper sheet. The Rogers layers were used for printing Curl and HIS because it offers low RF loss compared to FR-4. This fivelayer stratified antenna design allows for a simple, efficient and cost-effective fabrication. The QACH is fed from the bottom using four SMA (SubMiniature version A) connectors (A1-A4) of radius $1.3 \mathrm{~mm}$.

Fig 1 (a) shows the QACH geometry where an HIS [16-20] metamaterial layer is inserted within the substrate layer. This allows for the antenna height to be reduced from $37.5 \mathrm{~mm}$ [15] to $17.2 \mathrm{~mm}\left(\lambda_{1.597} / 10.9\right)$. Fig. 2 . shows the HIS unit model where its height from the ground plane is defined as $\mathrm{x}$. Using established theory, the initial HIS unit model was optimized such that the zeroreflection phase point to occur at $1.597 \mathrm{GHz}$ and covers the required bandwidth for a reflection phase of $+/-90^{\circ}$. This was achieved where $x=5.82 \mathrm{~mm}$ (Fig 2. (b)). However, when placed in the QACH element it was found that the antenna reflection coefficient bandwidth was not meeting the necessary requirements. Using an EM simulator optimizer, the height of HIS was varied to achieve the desired impedance bandwidth. The required steady state was accomplished where $\mathrm{x}=9.66 \mathrm{~mm}$.

Typically, the zero-reflection phase point is calculated normal to the surface and not at an oblique angle. However, the QACH has a tilted beam (i.e., at an oblique angle) not normal to the HIS surface leading to the final optimal value of $\mathrm{x}$ which was higher in comparison with the initial theoretical value.

Fig. 3 shows the surface currents of the antenna element. It can be seen that the primary current component is confined to the excited curl arm. However, lower amplitude current is also present on the other three passive curls and on the HIS metal patches. These all contribute to radiation for producing a circularly polarized tilted beam [15].

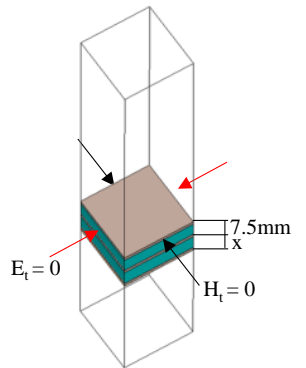

(a)

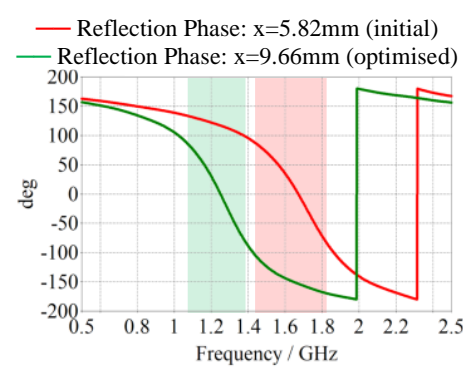

(b)
Fig. 2. (a) Initial HIS unit model. (b) The resultant reflection phase diagram. The initial theoretical design where $\mathrm{x}=5.82 \mathrm{~mm}$ showing good phase matching for radiation normal to the surface. Optimization within QACH element done to provide better performance for the tilted beam application, resulting in $\mathrm{x}$ becoming $9.66 \mathrm{~mm}$.
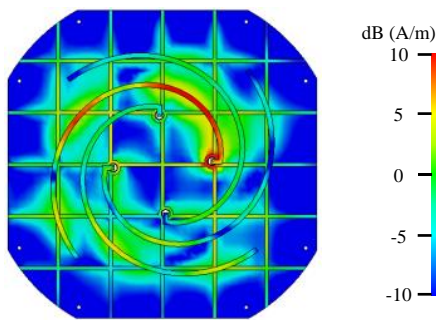

Fig. 3. The average surface currents over the $\mathrm{QACH}$ antenna element at the center frequency $1.597 \mathrm{GHz}$.

In this configuration, the antenna produces a tilted beam with a maximum gain of $8 \mathrm{dBic}$ at $\theta_{\max }=40^{\circ}$. All simulations in this paper are performed using the Dassault Systems Computer Simulation Technology (CST) software [21].

Each element consists of four curl arms [22], each fed from a port at the beginning of the curl (Fig. 1 (b)). Excitation of each port produces a circularly polarized tilted beam covering a quadrant [16]. The antenna provides a tilted beam of right-hand circular polarization over a wide bandwidth, as explained in [15] and [22].

\section{ELEMENT'S RESULTS}

The reflection coefficient measurements are taken with a Rohde \& Schwarz ZNA40 four port VNA [23]. Each port is measured individually by connecting a single port to the VNA and leaving the remaining ports open. The antenna is able to operate both when the remaining ports are open-circuited or $50 \Omega$ terminated. Opencircuit conditions have been chosen in the final design to provide higher efficiency. When a single port is excited and the remaining ports are open-circuited, the non-excited curl arms act as parasitic elements and so are not included in the scattering matrix, hence port isolation is only applicable when the element is placed in an array in Section IV. In final product integration, open-circuit Single Pole 4 Throw (SP4T) switches will be used for port selection. The measured and simulated reflection coefficients in Fig. 4 show that the measured reflection coefficient is below $-9 \mathrm{~dB}$ for all ports within the targeted band. Similar to many cellular communication 
devices [24, 25], a reflection coefficient better than $-6 \mathrm{~dB}$ can be sufficient to provide the required performance. In here, by maintaining a reflection coefficient less than $-9 \mathrm{~dB}$ and an antenna array gain greater than $6.6 \mathrm{dBic}$ at all angles, we are able to meet the link budget requirements of Inmarsat Class 12 [5]. If necessary, the reflection coefficient could be further expanded by improved matching and bringing the second resonance at $1.74 \mathrm{GHz}$ to a lower frequency. However, this may require a slightly greater antenna height. While there is a small shift in the resonant frequency between measurement and simulation, the results are in good agreement and both cover the required bandwidth.

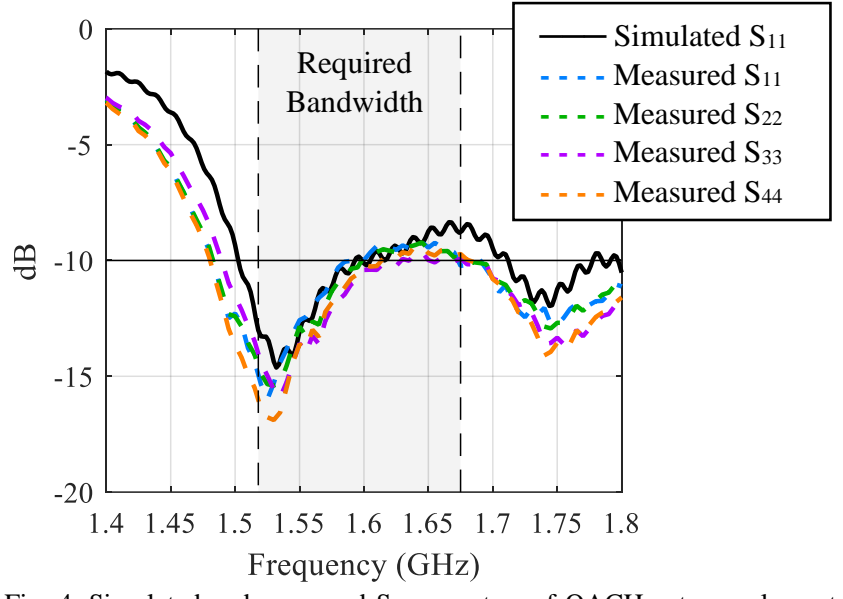

Fig. 4. Simulated and measured S-parameters of QACH antenna element shown in Fig. 1.

The element radiation patterns were measured with a SATIMO/MVG StarLab testing chamber [26] and are shown in Fig. 5. together with those captured in simulations. The results confirm that the quad-arm curl antenna generates an RHCP tilted beam with an RHCP gain greater than $8 \mathrm{dBic}$. The tilt of the beam is maintained at $\theta=40^{\circ}$ at all test frequencies. The axial ratio is maintained below $8 \mathrm{~dB}$ across the $3 \mathrm{~dB}$ beam width for all frequencies. The requirements for the target Inmarsat BGAN class 12 only necessitate an axial ratio below $8 \mathrm{~dB}$ for its ground terminal applications [10]. It is possible for a curl antenna and HIS to have a further improved axial ratio, however this would require increasing the height of the antenna substrate. Here we have optimized the antenna to reduce its height and in turn, its weight as far as possible while meeting Inmarsat BGAN Class 12 link requirements.

\section{IV. $1 \times 3$ QACH ANTENNA ARRAY}

Previously, [27] has developed a C-band $2 \times 2$ quad-arm curl antenna array, albeit without the key size benefits of an HIS and the vital low-cost implementation presented here. An L-band $2 \times 2$ QACH array configuration would be physically sizable $(332 \times 332$ $\mathrm{mm}$ ) even with the application of an HIS. Therefore, in order to reduce size and cost, a $1 \times 3$ rotated linear array has been considered, as shown in Fig. 6 (a) and (b) where antenna elements are physically rotated along directions $-30^{\circ}, 0^{\circ}$ and $30^{\circ}$. The rotation results in a total of 12 beams distributed evenly across the azimuth plane with $30^{\circ}$ separation. By combining each element beam with its two adjacent beams the directive gain increases and, with a phase shift applied across the array, precision beam steering can be achieved. Using this hybrid switched beam and phased array method, Fig. 7, the array can uniformly cover the entire azimuth plane despite its linear topology. With this method, we have been able to achieve wide beam steering while only requiring 3 HPAs, 3 LNAs, 4 phase shifters and 3 switches.

\section{A. Array Design}
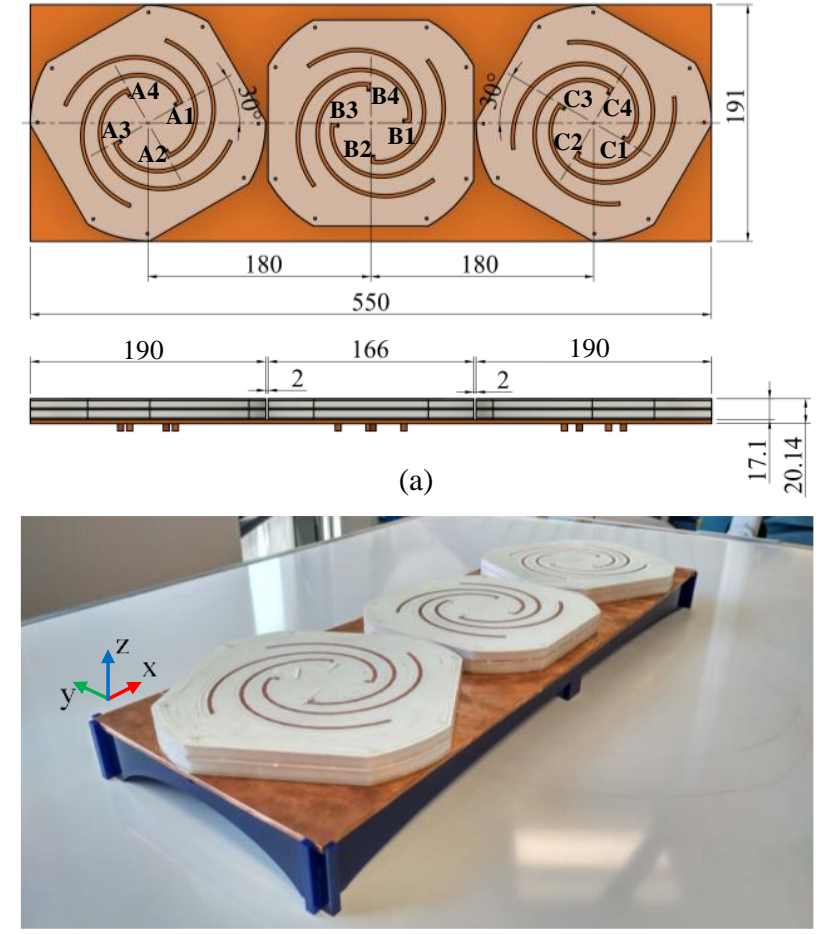

(b)

Fig 6. QACH array: (a) $1 \times 3$ linear array design (units in $\mathrm{mm}$ ); (b) array prototype.

In operation, three adjacent ports (1 port from each element, e.g. A1, B1 and C1) are selected with Single Pole 4 Throw (SP4T) switches. Phase shifters placed before each switch are used for precise beam forming for the selected ports. An example of how we have achieved a combined array beam is shown in Fig. 8. In this example, a beam is targeted along $\theta=70^{\circ}, \phi=0^{\circ}$. In order to target these coordinates, ports $\mathrm{A} 1, \mathrm{~B} 1$ and $\mathrm{C} 1$ are excited with equal amplitudes and phases of $-20^{\circ}, 0^{\circ}$ and $50^{\circ}$ respectively.

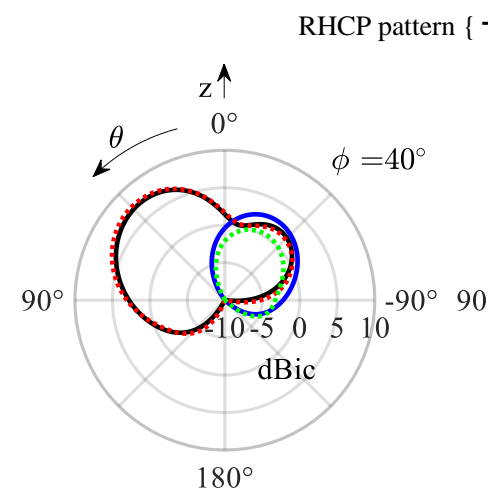

(a)

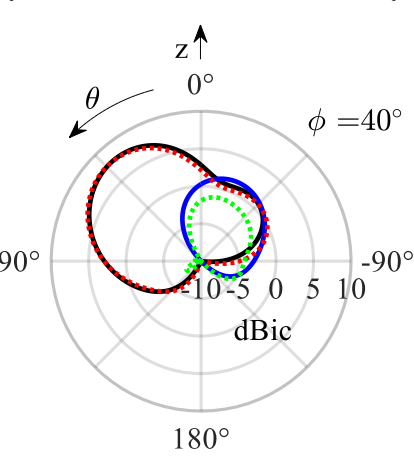

(b)

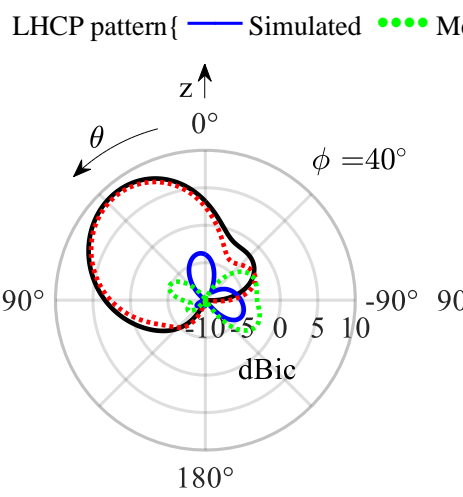

(c)

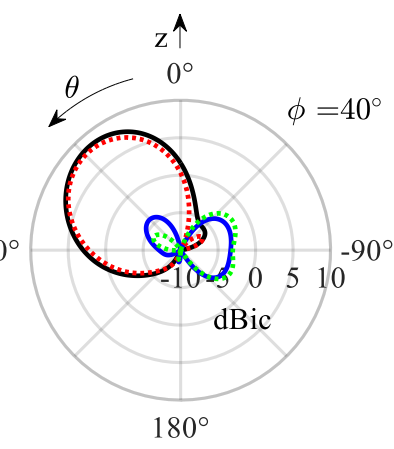

(d)

Fig. 5. Simulated and measured RHCP and LHCP radiation patterns of QACH antenna element at (a) $1.518 \mathrm{GHz}$, (b)1.559 GHz, (c)1.627 GHz and (d) 1.675 $\mathrm{GHz}$ over the operating frequency band. 


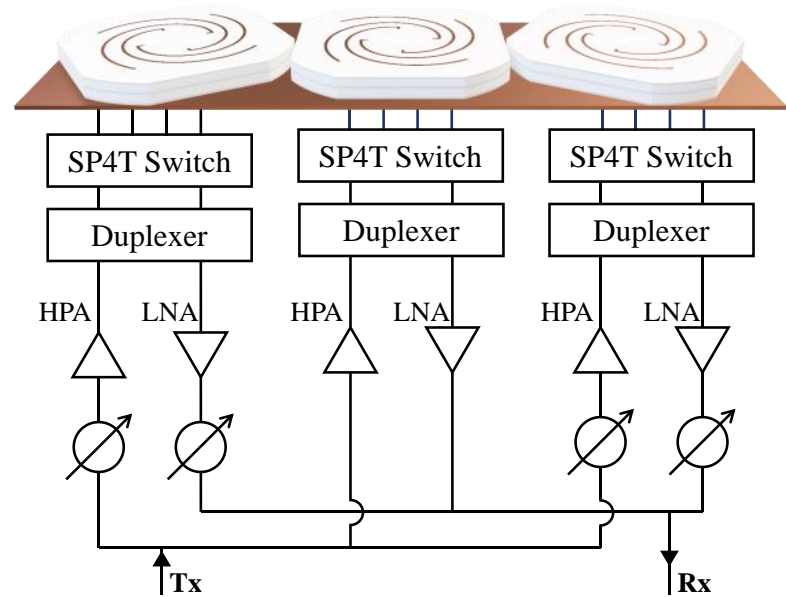

Fig. 7. Diagram of the simple, low-cost feeding network for the $1 \times 3 \mathrm{QACH}$ array.

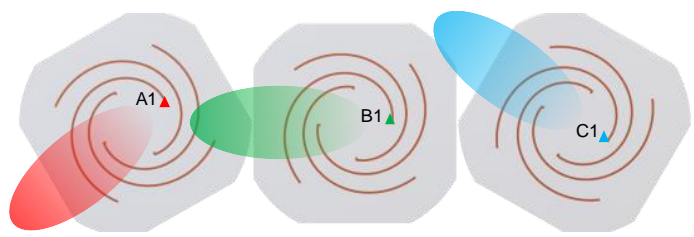

e.g. Beam $1=\mathrm{A} 1_{1<-20}+\mathrm{B} 1_{1<0}+\mathrm{C} 1_{1<50}$ $=1 \angle-20+1 \angle 0+1 \angle 50$

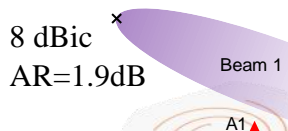

$\downarrow$

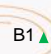

C1.

Fig. 8. Example diagram of the hybrid beam-steering method.

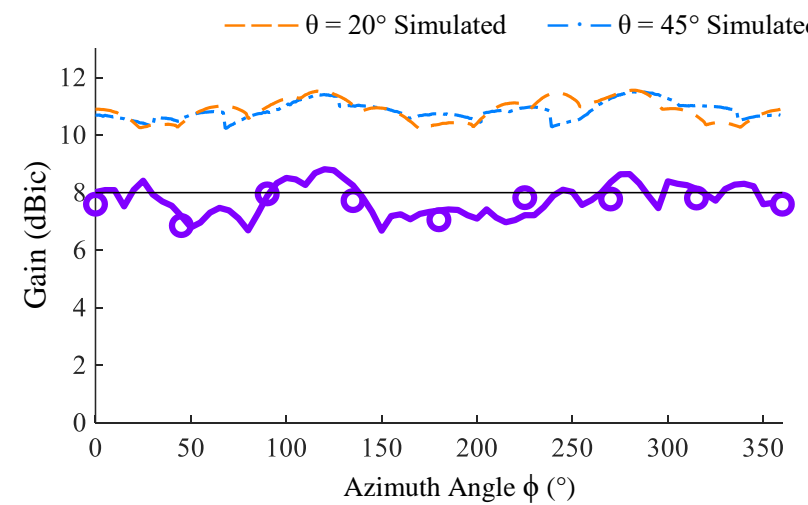

(a) $1.538 \mathrm{GHz}\left(\mathrm{Rx}_{\text {center }}\right.$

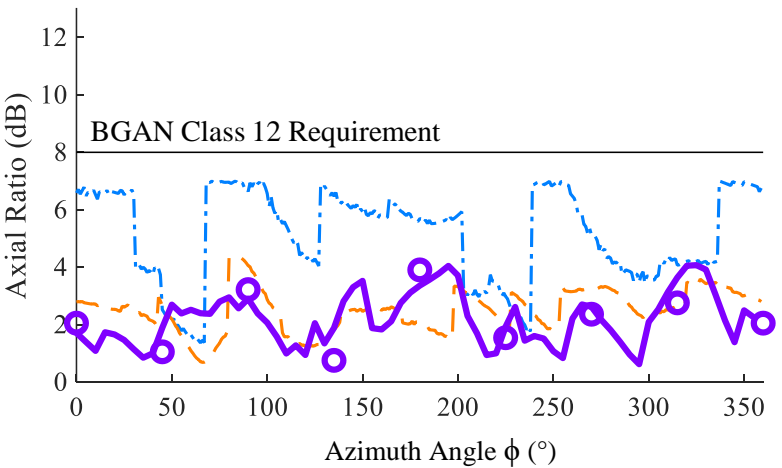

(c) $1.538 \mathrm{GHz}\left(\mathrm{Rx}_{\text {center }}\right)$

\section{B. Array Performance Verification}

The antenna array has been measured in a NSI-MI Technologies and Frequency's near-field anechoic test chamber at DISC [28]. A bespoke feeding network, Fig. 9, controls both the phase shifting and port switching. This RF board uses commercially available components (e.g. MACOM MAPS-010163, Mini-Circuits CMA545G1+, Analogue Devices ADRF5047) and follows a broadly similar scheme to that shown in Fig 7.

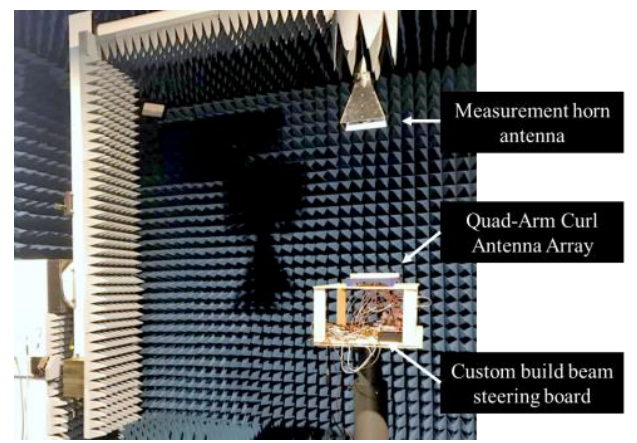

Fig. 9. QACH array performance test setup.

Fig. 10 (a) and (b) show the azimuth scanning performance of the proposed array at elevation angles of $20^{\circ}, 45^{\circ}$ and $70^{\circ}$. The average gain at $\theta=70^{\circ}$ is $7.96 \mathrm{dBic}$ and ranges from $6.63 \mathrm{dBic}$ to $12.78 \mathrm{dBic}$ for the three elevation angles shown. Some reduction in gain can be seen around $\phi=0^{\circ}$ and $\phi=180^{\circ}$. This is due to the linear array topologies and asymmetrical placement of antenna elements. The measured results at $70^{\circ}$ elevation are close to the simulated results for both the Rx and Tx bands.

The axial ratio of the respective beams is shown in Fig. 10 (c) and (d). The axial ratio remains below the required $8 \mathrm{~dB}$ for the majority of the beams. The measured axial ratio for the beams at $\theta$ $=70^{\circ}$ matches the simulated results well, showing very little variation for most points.

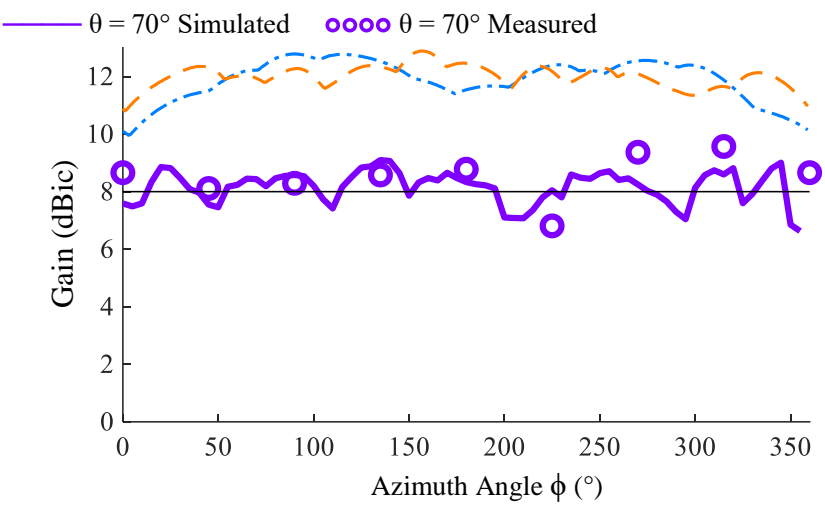

(b) $1.651 \mathrm{GHz}\left(\mathrm{Tx}_{\text {center }}\right)$

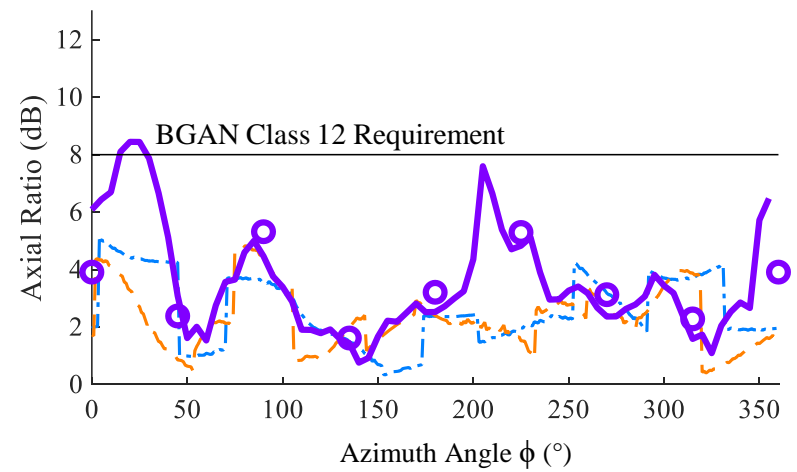

(d) $1.651 \mathrm{GHz}\left(\mathrm{Tx}_{\text {center }}\right)$

Fig. 10. Beam steering performance (predicted \& measured) of the $1 \mathrm{x} 3 \mathrm{QACH}$ array: (a) \& (b) gain and (c) \& (d) axial ratio at $1.538 \mathrm{GHz} \& 1.651 \mathrm{GHz}$. 
RHCP pattern $\{-$ Simulated $\cdots \bullet$ Measured $\}$
LHCP pattern $\{-$ Simulated $\cdots$ Measured $\}$
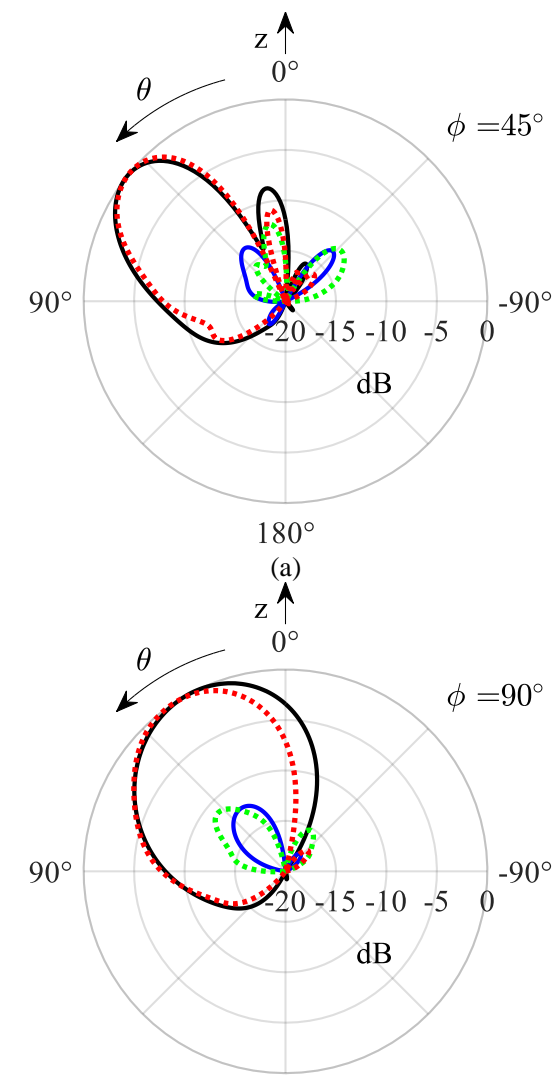

$180^{\circ}$

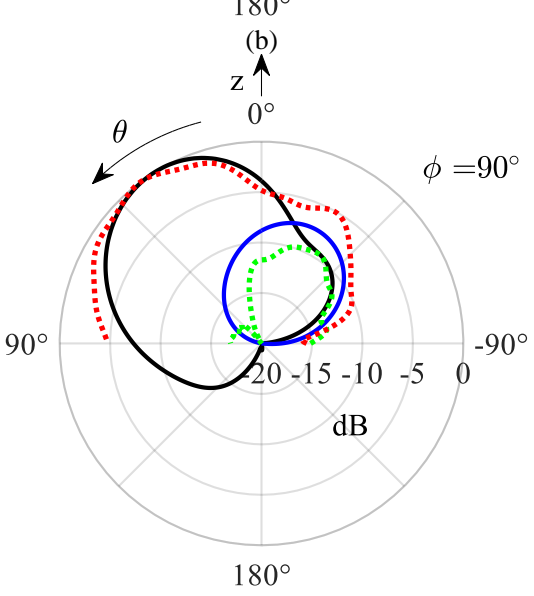

(c)

Fig. 11. Simulated and measured RHCP and LHCP polar patterns showing the array steering performance for $\mathrm{Tx}$ and $\mathrm{Rx}$ beam maximum. (a) Tx beam targeting $(\theta, \phi)=\left(70^{\circ}, 45^{\circ}\right)$; (b) Tx beam targeting $(\theta, \phi)=\left(45^{\circ}, 90^{\circ}\right) \&(\mathrm{c})$ Rx beam targeting $(\theta, \phi)=\left(45^{\circ}, 90^{\circ}\right)$.

Fig 11. shows the measured and simulated RHCP and LHCP radiations patterns of the array steering when a beam targeting in the direction of $(\theta, \phi)=\left(70^{\circ}, 45^{\circ}\right)$ and $\left(45^{\circ}, 90^{\circ}\right)$ for receive and transmit frequency bands. As the receive band does not causes interference, the back lobes are non-concerning for the satellite link. In transmit mode, the RHCP back lobe obtained was found to be better than $-9 \mathrm{~dB}$ in the majority of cases. The measured radiation patterns show good agreement with the simulated data.

The S-parameters of the array have been measured with an R\&S ZVA40 VNA. In operation, as explained previously, only three ports in the array (one port in each element) are in use at any one time while the other nine ports are left open-circuited. Therefore, when measuring the reflection coefficient and isolation between ports, only three ports are used per measurement.
Fig. 12 shows the S-parameters measured for all 12 ports (e.g $\mathrm{S}_{\mathrm{A} 1, \mathrm{~A} 1}-\mathrm{S}_{\mathrm{C} 4, \mathrm{C} 4}$ ) with unexcited ports left open circuited. The measured reflection coefficients remain below $-9 \mathrm{~dB}$ for the required frequency band, as anticipated by the simulation. The isolation between ports remains below $-20 \mathrm{~dB}$ for all ports. The radiation efficiency remains above $98 \%$ for both single port and array configurations. The total efficiency of the array remains above $90 \%$.

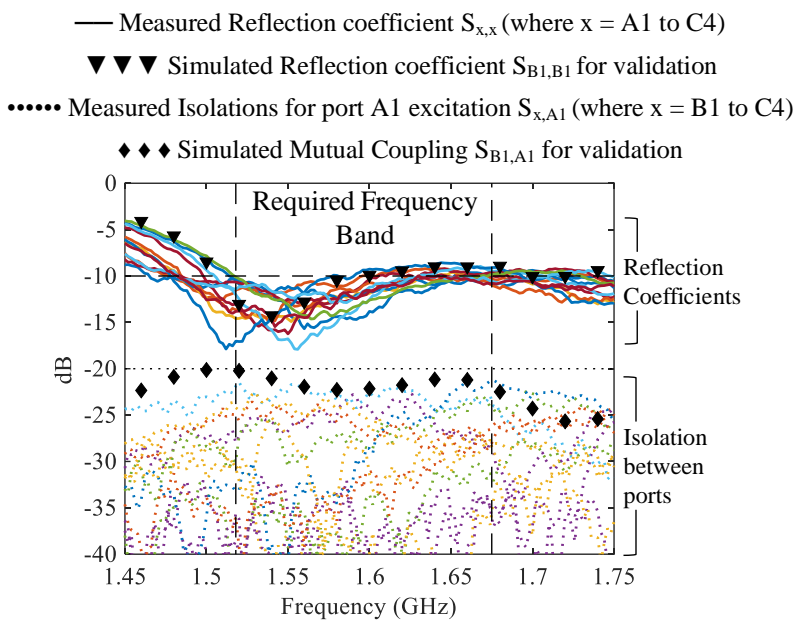

Fig. 12. Array reflection coefficients and isolation between the 12 ports.

\section{CONCLUSION AND DisCUSSION}

A $1 \times 3$ quad-curl antenna with an HIS substrate in a linear array configuration has been presented for L-band satellite communications. The QACH antenna unit element provides an operating bandwidth of greater than $10 \%(1.518-1.675 \mathrm{GHz})$, allowing for shared-aperture functionality. The height of the antenna has been reduced by the application of an HIS metamaterial layer. As a result, it is only $17.2 \mathrm{~mm}$ in height $\left(\lambda_{1.597 / 10.9)}\right.$. The proposed array can provide RHCP beam steering within elevation $20^{\circ}<\theta<70^{\circ}$ and full azimuth coverage, while still maintaining an average gain of $7.96 \mathrm{dBic}$ at $\theta=70^{\circ}$. In addition, as the proposed antenna array only consists of three elements, the feeding network requires only 4 phase shifters, 3 duplexers, 3 switches, 3 HPAs, and 3 LNAs. In comparison with a typical $3 \times 3$ patch array, this small number of components significantly reduces the complexity and cost of the array feeding network.

Table 1. A comparison between this work and existing works.

\begin{tabular}{|l|c|c|c|c|}
\hline & $\begin{array}{c}\text { This } \\
\text { work }\end{array}$ & {$[12]$} & {$[13]$} & {$[14]$} \\
\hline Array Type & Linear & Planer & Linear & Planer \\
\hline $\begin{array}{l}\text { Elevation } \\
\text { Steering Angles }\end{array}$ & $+/-70$ & $+/-60^{\circ}$ & $+/-90$ & $+/-60^{\circ}$ \\
\hline Bandwidth & $10 \%$ & $43 \%$ & $5 \%$ & $3.5 \%$ \\
\hline $\begin{array}{l}\text { Full Azimuth } \\
\text { Coverage }\end{array}$ & Yes & Yes & No & Yes \\
\hline Thickness & $0.09 \lambda$ & $0.17 \lambda$ & $0.04 \lambda$ & $0.04 \lambda$ \\
\hline $\begin{array}{l}\text { RF Network } \\
\text { Cost }\end{array}$ & Low & High & Medium & High \\
\hline $\begin{array}{l}\text { Circular } \\
\text { Polarization }\end{array}$ & Yes & No & No & No \\
\hline
\end{tabular}

A complete comparison between the proposed antenna array design and a number of similar works [12-14] is shown in Table 1. The work presented in this paper is shown to provide an advantageous combination of functionality that uniquely makes it ideal for low-cost GEO satellite applications. Because of the arrays low cost, small size, and high gain at angles near to the horizon, it delivers an innovative solution for on-the-move satellite IoT 
applications. Future systems with such a flat panel curl array will be able to provide mass deployable, uninterrupted internet access to market segments that would have previously found satellite communication impractical or unaffordable.

This work has been undertaken primarily for the BGAN Class 12 requirements. Yet, the QACH is easily up-scalable to $18 \mathrm{GHz}$ while maintaining low-cost manufacturing. Hence, with the arrival of 5G multiband, the work presented here has a variety of novel space and terrestrial communication applications beyond the Lband demonstrated in this work.

\section{REFERENCES}

[1] "Unleashing cellular connectivity for IoT applications," TATA Communications, [Online] Available:

https://www.tatacommunications.com/wpcontent/uploads/2019/07/Whitepaper-Unleashing-cellularconnectivity-for-IoT-applications.pdf Accessed on Dec. 10, 2019

[2] "Connected nations 2018: UK report," Ofcom, London, United Kingdom. Dec. 18, 2019. [Online]. Available: https://www.ofcom.org.uk/_data/assets/pdf_file/0020/130736/Con nected-Nations-2018-main-report.pdf.

[3] "Inmarsat BGAN for land," Inmarsat plc. London, United Kingdom. [Online] Available: https://www.inmarsat.com/wpcontent/uploads/2015/06/Inmarsat_BGAN_For_Land_USGov_Apri 1_2014_EN_LowRes.pdf Accessed on: Dec. 12, 2019.

[4] "The BGAN coverage map," Ground Control. California, US [Online] Available: https://www.groundcontrol.com/bgan_coverage_map.htm, Accessed on Apr. 14, 2020.

[5] "Cobham EXPLORER 323 and EXPLORER 6075LX terminals receive Inmarsat type approval," Inmarsat plc. London, United Kingdom. [Online] Available: https://www.inmarsat.com/en/news/latestnews/enterprise/2020/cobham-explorer-323-and-explorer-6075lxterminals-receive-inmarsat-type-approval.html Accessed on: Dec. $15,2020$.

[6] B. Schneiderman, "The next wave: low earth orbit constellations," Los Angeles, USA, Mar. 4, 2019. [online] Available: http://satellitemarkets.com/news-analysis/next-wave-low-earthorbit-constellations, Accessed on Dec. 15, 2019.

[7] H. Bayer, A. Krauss, T. Zaiczek, R. Stephan, O. Enge-Rosenblatt, and M. A. Hein. "Ka-band user terminal antennas for satellite communications," IEEE Antennas Propag. Mag., vol 58, pp. 76-88, Feb. 2016.

[8] A. V. Shishlov, "Vehicular antennas for satellite communications," 2011 VIII Intern. Conf. Antenna Theory and Techn., Kyiv, 2011, pp. 34-39.

[9] J. Huang, "L-band phased array antennas for mobile satellite communications," 37th IEEE Vehic. Technol. Conf., Tampa, Florida, USA, 1987, pp. 113-117.

[10] Z. Wang, S. Fang, S. Fu and S. Jia, "An Inmarsat BGAN terminal patch antenna array with unequal input impedance elements and conductor-backed ACPW series-feed network," in IEEE Trans. Antennas Propag., vol. 60, no. 3, pp. 1642-1647, March 2012.

[11] N. B. Buchanan, V. F. Fusco, M. van der Vorst and D. Zelenchuk, "A circular polarized self-tracking L band array with high bandwidth and scan beamwidth for Inmarsat BGAN applications," Proc. of the 5th Eur. Conf. on Antennas Propag. (EUCAP), Rome, 2011, pp. 211215.

[12] J. A. Kasemodel, C. C. Chen, and J. L. Volakis, "Wideband planar array with integrated feed and matching network for wide-angle scanning," IEEE Trans. Antennas Propag., vol. 61, no. 9, pp. 45284537, Jun. 2013

[13] R. Wang, B.-Z. Wang, X. Ding, and X.-S. Yang, "Planar phased array with wide-angle scanning performance based on image theory," IEEE Trans. Antennas Propag., vol. 63, no. 9, pp. 39083917, Jun. 2015.

[14] N. Haider, A. G. Yarovoy, and A. G. Roederer, "L/S-band frequency reconfigurable multiscale phased array antenna with wide angle scanning," IEEE Trans. Antennas Propag., vol. 65, no. 9, pp. 45194528, Jul. 2017.

[15] H. Zhou, A. Pal, A. Mehta, D. Mirshekar, and H. Nakano, "A fourarm circularly polarized high-gain high-tilt beam curl antenna for beam steering applications," IEEE Antennas Wireless Propag. Letters, vol 17, No. 7, pp. 1034 - 1038. Jun. 2018.

[16] H. Nakano, K. Hitosugi, N. Tatsuzawa, D. Togashi, H. Mimaki, and J. Yamauchi, "Effects on the radiation characteristics of using corrugated reflector with a helical antenna and an electromagnetic band-gap reflector with a spiral antenna," IEEE Trans. Antennas Propag., vol 53, No 1, pp. 191-199, Jan 2005.

[17] F. Yang, and Y. Rahmat-Samii, "A low-profile circularly polarized curl antenna over an electromagnetic bandgap (EBG) surface," Microw. Opt. Technol. Lett., vol 31, No. 4, Nov 2001

[18] P. Deo, A. Mehta, D. Mirshekar-Syahkal, P. J. Massey and H. Nakano, "Thickness reduction and performance enhancement of steerable square loop antenna using hybrid high impedance surface," IEEE Trans. Antennas Propag., vol. 58, no. 5, May 2010.

[19] P. Deo, A. Mehta, D. Mirshekar-Syahkal and H. Nakano, "An HISbased spiral antenna for pattern reconfigurable applications," in IEEE Antennas Wireless Propag. Lett., vol. 8, pp. 196-199, 2009

[20] W. Zhang, A. Pal, A. Mehta, D. Mirshekar-Syahkal and H. Nakano, "Low profile pattern-switchable multibeam antenna consisting of four L-shaped microstrip lines," in IET Microwaves, Antennas Propag., vol. 12, no. 11, pp. 1846-1851, 1292018

[21] "CST studio suit," Dassault Systèmes SE, Vélizy-Villacoublay, France. Available: https://www.3ds.com/productsservices/simulia/products/cst-studiosuite/?utm_source=cst.com\&utm_medium=301\&utm_campaign=cs t. Accessed on Dec. 5, 2019.

[22] H. Nakano, S. Okuzawa, K. Ohishi, H. Mimaki and J. Yamauchi, "A curl antenna," in IEEE Trans. Antennas Propag., vol. 41, no. 11, pp. 1570-1575, Nov. 1993, doi: 10.1109/8.267357.

[23] "R\&S®ZVA vector network analyzers," Rohde \& Schwarz, Munich, Germany. [Online]. Available: https://www.rohdeschwarz.com/uk/product/zva-productstartpage_63493-9660.html. Accessed on Dec. 10, 2019.

[24] C. Hsu and S. Chung, "Compact multiband antenna for handsets with a conducting edge," in IEEE Trans. on Antennas Propag., vol. 63 , no. 11, pp. 5102-5107, Nov. 2015, doi: 10.1109/TAP.2015.2473657.

[25] C. Huang, Y. Jiao, Z. Weng and X. Li, "A planar multiband antenna based on CRLH-TL ZOR for 4G compact mobile terminal applications," 2018 Intern. Workshop Antenna Technol., Nanjing, 2018, pp. 1-3, doi: 10.1109/IWAT.2018.8379200.

[26] "MVG Star Lab 650-1800 MHz," Microwave Vision Group. [Online] Available: https://www.mvgworld.com/en/products/field_product_family/antennameasurement-2/starlab. Accessed on Oct. 19, 2018.

[27] H. Zhou, A. Pal, A. Mehta, H. Nakano, A. Modigliana, T Arampatzis, and P. Howland. "Reconfigurable phased array antenna consisting of high-gain high-tilt circularly polarized four-arm curl elements for near horizon scanning satellite applications," IEEE Antennas Wireless Propag. Lett., vol. 17, no. 12, pp $2324-2328$. Dec. 12,2018

[28] "Disruptive innovation for space centre brochure," Satellite Applications Catapult, United Kingdom [Online]. Available: https://sa.catapult.org.uk/wp-content/uploads/2018/10/C224868DISC-Brochure-ONLINE-.pdf, Accessed on: Dec. 11, 2019. 